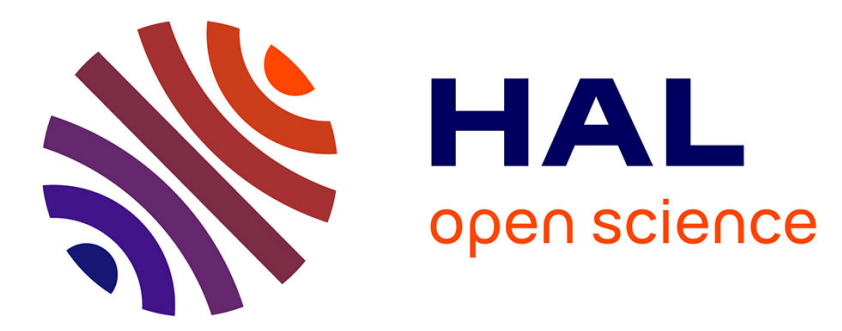

\title{
Competition between wireless service providers sharing a radio resource
}

\author{
Patrick Maillé, Bruno Tuffin, Jean-Marc Vigne
}

\section{To cite this version:}

Patrick Maillé, Bruno Tuffin, Jean-Marc Vigne. Competition between wireless service providers sharing a radio resource. 11th International Networking Conference (NETWORKING), May 2012, Prague, Czech Republic. pp.355-365, 10.1007/978-3-642-30054-7_28. hal-00725181

\section{HAL Id: hal-00725181 \\ https://hal.science/hal-00725181}

Submitted on 24 Aug 2012

HAL is a multi-disciplinary open access archive for the deposit and dissemination of scientific research documents, whether they are published or not. The documents may come from teaching and research institutions in France or abroad, or from public or private research centers.
L'archive ouverte pluridisciplinaire HAL, est destinée au dépôt et à la diffusion de documents scientifiques de niveau recherche, publiés ou non, émanant des établissements d'enseignement et de recherche français ou étrangers, des laboratoires publics ou privés. 


\title{
Competition between wireless service providers sharing a radio resource
}

\author{
Patrick Maillé ${ }^{1}$, Bruno Tuffin ${ }^{2}$, and Jean-Marc Vigne ${ }^{2}$ \\ 1 Institut Telecom; Telecom Bretagne \\ 2 rue de la Châtaigneraie CS 17607 \\ 35576 Cesson Sévigné Cedex, France \\ patrick.maille@telecom-bretagne.eu \\ 2 INRIA Rennes - Bretagne Atlantique \\ Campus universitaire de Beaulieu \\ 35042 Rennes Cedex, France \\ btuffin@irisa.fr \\ jvigne@irisa.fr
}

\begin{abstract}
We present a model of competition on prices between two telecommunication service providers sharing an access resource, which can for example be the same WiFi spectrum. We obtain a two-level game corresponding to two time scales of decisions: at the smallest time scale, users play an association game by choosing their provider (or none) depending on price, provider reputation and congestion level, and at the largest time scale, providers compete on prices. We show that the association game always has an equilibrium, but that several can exist. The pricing game is then solved by assuming that providers are riskaverse and try to maximize the minimal revenue they can get at a user equilibrium. We illustrate what can be the outcome of this game and that there are situations for which providers can co-exist.
\end{abstract}

Keywords: Game Theory, wireless networks, pricing, shared spectrum, Wardrop equilibrium

\section{Introduction}

Internet access has become almost compulsory in everyday life, and each user has to decide through which provider to access the network. While there are areas with a single access point and provider, in most cases the Internet access has become highly competitive and users can choose the provider they prefer depending on a combination of price, offered quality of service (QoS), and reputation. Studying the outcome of the association choices from users is of major importance for providers, in order to decide if they have an interest in competing for the access offers, and if so, to define at which price.

We propose in this paper to study such a problem by means of non-cooperative game theory [1]. We define a two-level game where, at the largest time scale, providers set their price in order to maximize their revenue, and at the smallest 
time scale users choose the provider they are going to be associated with. This association choice is based on a perceived price which depends for each provider on the real price charged and a congestion cost pondered by the provider's reputation. A complication is that user choices have an impact on the QoS (i.e., the experienced congestion) of others, hence a non-cooperative game between users. At this level, we rather represent here a total demand level (the granularity of a unit of demand being infinitesimal) and the equilibrium notion follows Wardrop's principle, as classically done when dealing with transportation networks [2]. Even if providers decide their price before the association game is played, we assume and believe that they play smartly by anticipating the outcome of the association game, but also trying to figure out the reaction of their competitor which also tries to attract customers. This pricing game is then said to be a multiple-leader (the providers), multiple-follower (the users) game, that we analyze by backward induction.

This type of two-level game of direct competition between providers has already been studied in the literature. The most notable related papers $[3,4,5,6,7]$ usually deal with providers offering their own resource, and not shared as we do here, resulting in the most important novelty. The typical application we are looking at is a shared WiFi access. As an additional difference, even if as in $[3,4,5]$ QoS is represented by delay as we are going to do, we include a provider reputation characteristic. Also, several reputation-based models of WiFi network selection have already been studied in the literature $[8,9,10]$, but no price competition between providers is mentionned in those papers.

Demand is also assumed to be segmented to better differentiate the levels of reputation of providers among the population. Remark that a similar model with possible resource (i.e., spectrum) sharing (but without demand segmentation and provider reputation) has been introduced in [11], including an additional level of game on network investment for providers which could implement several technologies. Though, in [11], no pricing game equilibrium could exist with providers both using a resource, in contradiction to what is observed in practice. We show here that thanks to demand segmentation and providers reputation, such an equilibrium can exist with an access resource shared by providers.

The remaining of this paper is organized as follows. Section 2 presents the model: the demand segmentation and the two-level game we are considering and aiming to solve. Section 3 then describes how the users' association game at the smallest time scale can be solved, when access prices are fixed for each provider. We show that a user (Wardrop) equilibrium always exists, and that all perceived prices as well as total demand per segment at equilibrium are unique. Section 4 describes the upper-level pricing game between providers, that is played first with providers anticipating the resulting equilibria at the user level. Finally, conclusions and directions for future research are provided in Section 5. 


\section{Model}

We consider a set of wireless telecommunications service providers proposing a wireless access to users in a given geographical area. Any wireless technology can be proposed as long as the resource is shared between providers, a typical example being the 802.11 (WiFi) technology. All access points are modeled as a single common one accessible by all customers in the area, as is often the case in WiFi hotspots. The goal of each wireless operator is to maximize its revenue by choosing the best price per second, whereas the goal of users is to choose the operator proposing the best compromise between price, reputation, and congestion.

We denote by $\mathcal{O}$ the set of all providers. On a quite large time scale, each provider $o \in \mathcal{O}$ proposes an offer that we model here by a single average price per second $p_{o}$ (in monetary units per second).

On a shorter time scale, users select which provider to connect to (if any), based on their preferences. We assume that users are segmented according to some trait (wireless service used, age ...), reflecting different perceptions of congestion with each provider, that can be interpreted as reputation effects. We denote by $\mathcal{S}$ the set of segments. Users make their decisions based on the socalled perceived price of the different possibilities, that encompasses both the price, congestion, and reputation aspects.

More precisely, we consider that congestion is perceived as a cost that is added to the subscription price $p_{o}$ paid to the selected provider. The congestion level, denoted by $\ell$, is assumed to be a continuous, positive and strictly increasing function depending on the total user throughput. For each segment, the congestion perception of users is influenced by the reputation of providers they have chosen, and we assume this dependence to be of multiplicative form in the congestion. So that if $\gamma_{o, s}$ is the reputation factor of Operator $o \in \mathcal{O}$ for segment $s \in \mathcal{S}$, the total perceived price $\bar{p}_{o, s}$ for a segment- $s$ user connecting to provider $o$ equals

$$
\bar{p}_{o, s}:=p_{o}+\gamma_{o, s} \ell\left(\sum_{\substack{i \in \mathcal{O} \\ t \in \mathcal{S}}} \lambda_{t} d_{i, t}\right),
$$

where $d_{o, s}$ is the volume of customers (seen as a continuous quantity) of segment $s$ on Operator $o$ and $\lambda_{s}$ is the average throughput of a segment- $s$ customer. Users are indeed assumed here non-atomic, i.e., the influence of an individual user is negligible, so that each user selects the provider with the lowest perceived price, without considering the congestion he may create. The total demand (or throughput) of segment $s$ is then $d_{s}=\sum_{o \in \mathcal{O}} d_{o, s}$, while at Operator $o$ it is $\sum_{s \in \mathcal{S}} d_{o, s}$.

Finally, for each population segment $s$, the aggregated demand $d_{s}$ is a function of the perceived price at its chosen provider(s), that is the smallest perceived price for that segment among all operators, $\min _{o} \bar{p}_{o, s}$. We call $D_{s}$ this function and suppose that it is upper-bounded, nonnegative, decreasing, continuous, and strictly positive at 0 . 


\section{Users' association game}

We describe in this section the expected outcomes of the game that is played among users. As described in the previous section, for a given price profile $\left(p_{1}, \ldots, p_{|\mathcal{O}|}\right)$, a stable situation -i.e., an equilibrium- is a user distribution among operators for each segment such that no user has an incentive to switch providers. This corresponds to the notion of a Wardrop equilibrium, which can be characterized mathematically as nonnegative demand values $\left(d_{o, s}\right)_{o \in \mathcal{O}, s \in \mathcal{S}}$ satisfying

$$
\left\{\begin{array}{r}
\forall o \in \mathcal{O} \quad \forall s \in \mathcal{S} \quad \bar{p}_{o, s}=p_{o}+\gamma_{o, s} \ell\left(\sum_{i \in \mathcal{O}, t \in \mathcal{S}} \lambda_{t} d_{i, t}\right) \\
\forall o \in \mathcal{O} \quad \forall s \in \mathcal{S} \quad d_{o, s}>0 \Longrightarrow \bar{p}_{o, s}=\min _{i \in \mathcal{O}}\left(\bar{p}_{i, s}\right) \\
\forall s \in \mathcal{S} \quad \sum_{i \in \mathcal{O}} d_{i, s}=D_{s}\left(\min _{i \in \mathcal{O}} \bar{p}_{i, s}\right) .
\end{array}\right.
$$

The definition of the perceived price is written in the first line. A necessary condition for a demand $d_{o, s}$ to be strictly positive is explained in the second line, reflecting the fact that only providers with cheapest perceived price are selected. The relation between the perceived price of each segment and its demand level is expressed in the last line.

Note that a solution of the system above always exists:

Proposition 1. For every price profile p, there exists a Wardrop equilibrium. Moreover, all the perceived prices $\bar{p}_{o, s}$ for each segment $s \in \mathcal{S}$ and each operator $o \in \mathcal{O}$ are unique, and all demand levels $\left(d_{s}\right)_{s \in \mathcal{S}}$ are also unique.

In addition, the set $W(p)$ of Wardrop equilibria corresponding to the price profile $p$ is compact.

Proof. The existence is a direct application of Theorem 5.4 in [12]. To prove the uniqueness of perceived prices, we define the function

$$
g(x):=\sum_{s \in \mathcal{S}} \lambda_{s} D_{s}\left(\min _{i \in \mathcal{O}} f_{i, s}(x)\right)-x,
$$

where $f_{i, s}(x)=p_{i}+\gamma_{i, s} \ell(x)$, that represents the difference between what the total demand should be as a response to the total throughput $x$, and $x$ itself. From (2), at a Wardrop equilibrium $g$ should be null. But since $g$ is continuous, strictly decreasing, positive at zero and negative at $+\infty, g$ has a unique zero $\hat{x}$. Consequently a Wardrop equilibrium total throughput verifies $\sum_{o \in \mathcal{O}, s \in \mathcal{S}} \lambda_{s} d_{o, s}=\hat{x}$ and is therefore unique.

Then the uniqueness of all perceived prices comes from (1), and the uniqueness of segment demands from the last line of (2). 
To prove the compactness of the set $W(p)$ of Wardrop equilibria, we remark that the system (2) is equivalent to the system

$$
\left\{\begin{array}{l}
\forall o \in \mathcal{O}, \forall s \in \mathcal{S}, \\
d_{o, s}\left(p_{o}+\gamma_{o, s} \ell\left(\sum_{i \in \mathcal{O}, t \in \mathcal{S}} \lambda_{t} d_{i, t}\right)-\min _{o^{\prime} \in \mathcal{O}}\left[p_{o^{\prime}}+\gamma_{o^{\prime}, s} \ell\left(\sum_{i \in \mathcal{O}, t \in \mathcal{S}} \lambda_{t} d_{i, t}\right)\right]\right)=0 \\
\forall s \in \mathcal{S}, \\
\sum_{i \in \mathcal{O}} d_{i, s}-D_{s}\left(\min _{o^{\prime} \in \mathcal{O}}\left[p_{o^{\prime}}+\gamma_{o^{\prime}, s} \ell\left(\sum_{i \in \mathcal{O}, t \in \mathcal{S}} \lambda_{t} d_{i, t}\right)\right]\right)=0 .
\end{array}\right.
$$

Therefore, Wardrop equilibria are demand values $d=\left(d_{o, s}\right)_{o \in \mathcal{O}, s \in \mathcal{S}}$ such that $F(d)=0$, for the continuous function $F:\left(\mathbb{R}^{+}\right)^{|\mathcal{O}| \times|\mathcal{S}|} \mapsto \mathbb{R}^{|\mathcal{S}|(|\mathcal{O}|+1)}$ with each coordinate given by the left-hand term of the equations in (3). Consequently, $W(p)$ is a closed subset of $\left(\mathbb{R}^{+}\right)^{|\mathcal{O}| \times|\mathcal{S}|}$. Since it is also bounded (due to the uniqueness of per-segment demands $\left.\left(d_{s}\right)_{s \in \mathcal{S}}\right), W(p)$ is compact.

While the existence of a Wardrop equilibrium is proved, there are situations where the equilibrium is not unique, as illustrated by the following example.

Example 1 Assume that we have only two operators, denoted by 1 and 2, competing on a single market segment $a$. We assume that the demand function is piecewise-linear and is defined by $D_{a}(x)=[5-x]^{+}$, where $[\cdot]^{+}=\max (\cdot, 0)$. Assume in addition that $\gamma_{1, a}=2, \gamma_{2, a}=1, \lambda_{a}=1$, and $\ell(x)=x$. Consider the operator price profile $\left(p_{1}, p_{2}\right)=(2,3)$. Then at a Wardrop equilibrium, $5-\min \left(\bar{p}_{1, a}, \bar{p}_{2, a}\right)=d_{1, a}+d_{2, a}$. Moreover $\bar{p}_{1, a}=2+2\left(d_{1, a}+d_{2, a}\right)$ and $\bar{p}_{2, a}=3+\left(d_{1, a}+d_{2, a}\right)$. Solving that system gives $d_{1, a}+d_{2, a}=1$ and $\bar{p}_{1, a}=\bar{p}_{2, a}=4$. In that case, the perceived prices are the same at each provider and users are indifferent between going to one provider or to the other, the congestion staying the same because the resource is shared, as soon as total demand is fixed to 1. Any $\left(d_{1, a}, d_{2, a}\right)=(u, 1-u)$ with $u \in[0,1]$ is a Wardrop equilibrium.

This example highlights the situation when several Wardrop equilibria can exist, that is when for some segment the minimal perceived price is the same at several providers: users in that segment are then indifferent between those providers because the congestion function and therefore the perceived prices will not change whatever the distribution.

\section{Pricing game between providers}

We now describe the competition between providers on prices. Each provider wants to maximize its revenue, but since several Wardrop equilibria may exist, several possible revenues may be associated to a price profile. A natural objective for an operator, that we consider in this paper, is to maximize the worst 
revenue it can expect. Formally, the minimal revenue of provider $o$ is expressed in monetary units per second, as

$$
R_{o}^{\min }(p):=p_{o} \times \min _{d \in W(p)} \sum_{s \in \mathcal{S}} d_{o, s}
$$

Note that the existence of the minimum is ensured by the compactness of $W(p)$, established in Proposition 1.

The equilibrium concept in this non-cooperative game between providers is the so-called Nash equilibrium [1]. A Nash equilibrium is a price profile such that no provider can increase his revenue by unilaterally changing his price. Formally, such a price equilibrium is therefore a price profile $\left(p_{1}^{*}, \ldots, p_{|\mathcal{O}|}^{*}\right)$ such that for every operator $i$ and every price profile $p_{i}, R_{i}^{\min }\left(p^{*}\right) \geq R_{i}^{\min }\left(p_{-i}^{*}, p_{i}\right)$.

For the rest of this article, we will analyze a duopoly. In order to determine the existence and uniqueness of a Nash equilibrium, we first compute the best-response function of each provider, that is, the function giving the profitmaximizing price value(s) for the considered provider, defined in terms of the price strategy of the opponent. Formally:

$$
\begin{aligned}
& \mathrm{BR}_{1}\left(p_{2}\right)=\arg \max _{p_{1} \geq 0} R_{1}^{\min }\left(p_{1}, p_{2}\right) \text { and } \\
& \operatorname{BR}_{2}\left(p_{1}\right)=\arg \max _{p_{2} \geq 0} R_{2}^{\min }\left(p_{1}, p_{2}\right) .
\end{aligned}
$$

Note that $R_{o}^{\min }$ is only piecewise continuous, thus it may happen that the arg max per se does not exist; in those cases we reasonably consider a price value giving a revenue close to the $\sup _{p_{o} \geq 0} R_{o}^{\min }$. The arg max above is therefore a slight abuse of notation.

A Nash equilibrium is simply a point $\left(p_{1}^{*}, p_{2}^{*}\right)$ for which $\mathrm{BR}_{1}\left(p_{2}^{*}\right)=p_{1}^{*}$ (or $p_{1}^{*} \in \mathrm{BR}_{1}\left(p_{2}^{*}\right)$ if it is made of several solutions) and equivalently $\operatorname{BR}_{2}\left(p_{1}^{*}\right)=p_{2}^{*}$. Graphically, if we draw the two best response curves on the same figure, the set of Nash equilibria is then the (possibly empty) set of intersection points of those curves. We now illustrate how this kind of equilibrium can be determined numerically with two operators.

Consider two operators numbered 1 and 2 in competition, and two market segments $a$ and $b$. Assume that $\gamma_{1, a}=\gamma_{2, b}=1.5$ and $\gamma_{1, b}=\gamma_{2, a}=0.04$. In addition, assume that $\lambda_{a}=1.0$ and $\lambda_{b}=0.02$. Finally, assume that the demand functions are $D_{a}(x)=[7-x]^{+}$and $D_{b}(x)=[9-x]^{+}$with $[\cdot]^{+}=\max (\cdot, 0)$, and that the congestion function is $\ell(x)=x$. The best response prices have been numerically computed, and are depicted in Figure 1 . One can readily check the existence and uniqueness of a Nash equilibrium for that instance. We also notice that the best-response curve of each operator is divided into two parts, with a discontinuity point because the segment choices at the Wardrop equilibrium change: for example, for $p_{1}=7$, the best strategy of Operator 2 is to set its price to attract only segment $a$ and maximize its revenue on that segment, whereas for $p_{1}>7.1$ it is better for Operator 2 to attract both segments, hence the discontinuity. Since the user segments choices in terms of preferred operators 
may vary, we display them as well in Figure 1 (as well as in subsequent figures): in the legend, we express user equilibrium choices of the form $\left(S_{1}, S_{2}\right)$, meaning that at the Wardrop equilibrium users of segments in $S_{1} \subset \mathcal{S}$ (respectively $S_{2} \subset \mathcal{S}$ ) choose Operator 1 (respectively Operator 2).

Remark also that at the Nash equilibrium $\left(p_{1}, p_{2}\right) \approx(4.45,3.45)$, each operator has some users, more exactly a full segment.

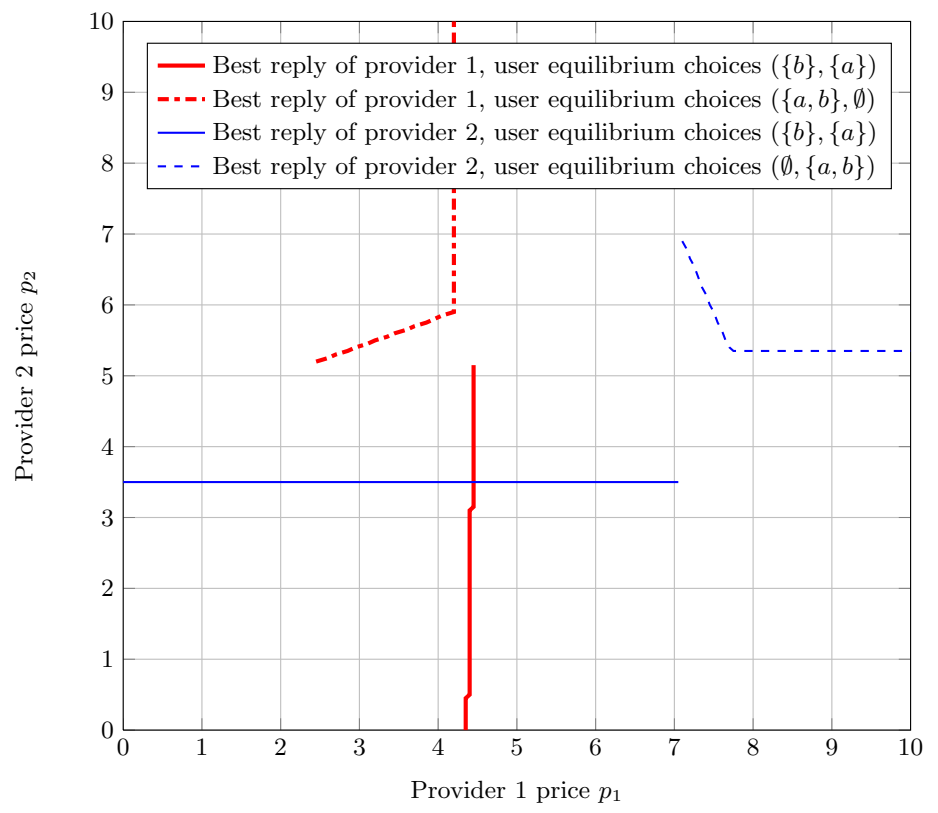

Fig. 1. Best-response curves of both operators.

To better explain the discontinuity of the best-response price $B R_{1}($.$) of$ provider 1 (a similar reason can be applied to the discontinuity on $B R_{2}($.$) ),$ we display in Figures 2 and 3 the revenue $R_{1}^{\min }\left(p_{1}, p_{2}\right)$ of Operator 1 in terms of $p_{1}$ for two values of $p_{2}, 5.1$ and 5.3 , located on both sides of the discontinuity point. It is clear from those figures that there are two local maxima (corresponding to two different segment choices at Wardrop equilibrium), and that the value of $p_{1}$ maximizing the revenue comes from a different local maximum.

If we look back again at Figure 1, we also observe piece-wise constant slopes in the best-response curves. To understand why, we plot in Figures 4 and 5 the revenue $R_{2}^{\min }\left(p_{1}, p_{2}\right)$ in terms of $p_{2}$ for $p_{1}=7.5$ and $p_{1}=8.0$. As it can be seen, for $p_{1}=7.5$, the maximum is obtained at the left extremity of the interval of $p_{2}$ such that the segment choices at Wardrop equilibrium are $\left(S_{1}, S_{2}\right)=(\emptyset,\{a, b\})$. But after around $p_{1}=7.75$, the maximum is obtained in the interior of the interval of $p_{2}$ such that the segment choices at Wardrop equilibrium are $(\emptyset,\{a, b\})$, which explains the slope change. 


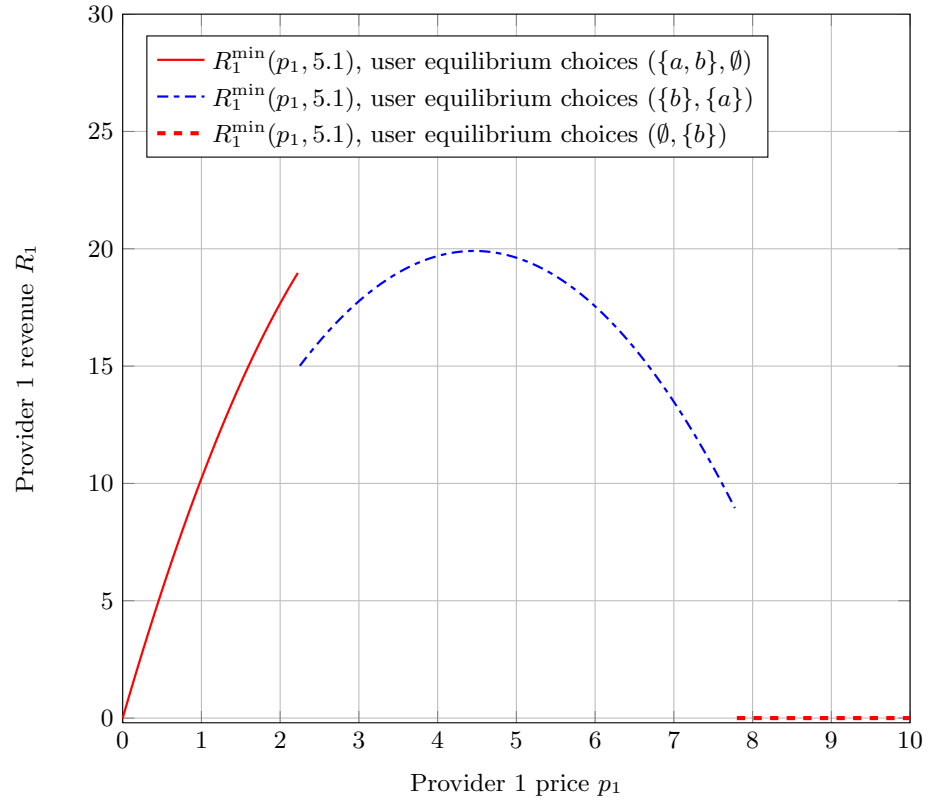

Fig. 2. Minimal revenue $R_{1}^{\min }\left(p_{1}, 5.1\right)$ in terms of $p_{1}$, with segment choices at Wardrop equilibrium.

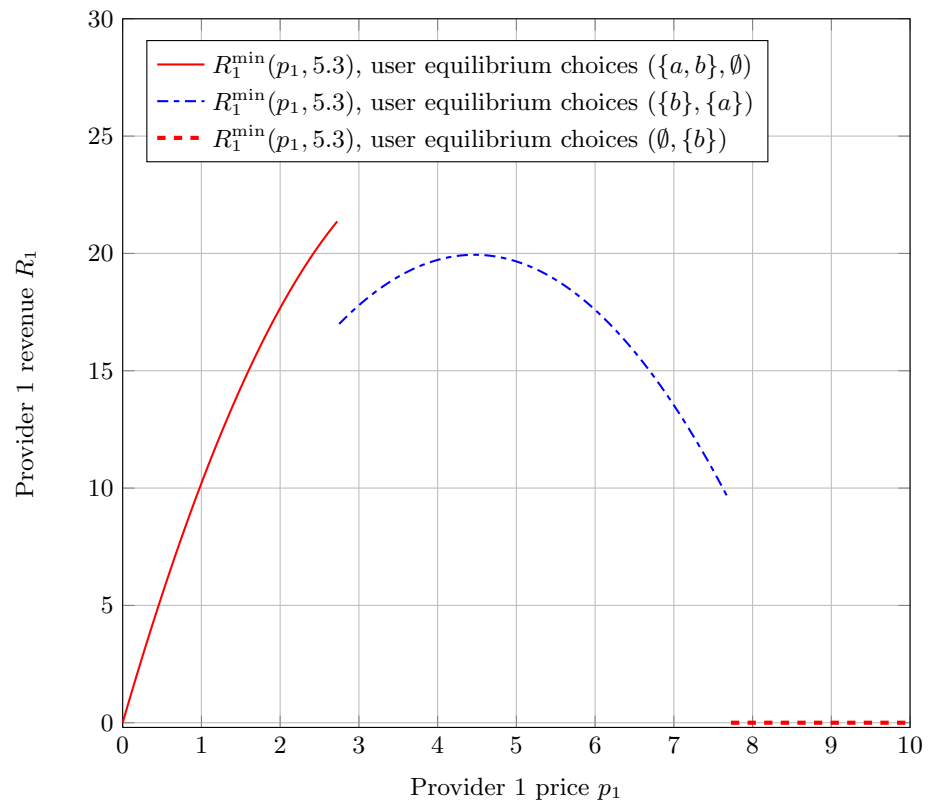

Fig. 3. Minimal revenue $R_{1}^{\min }\left(p_{1}, 5.3\right)$ in terms of $p_{1}$, with segment choices at Wardrop equilibrium. 


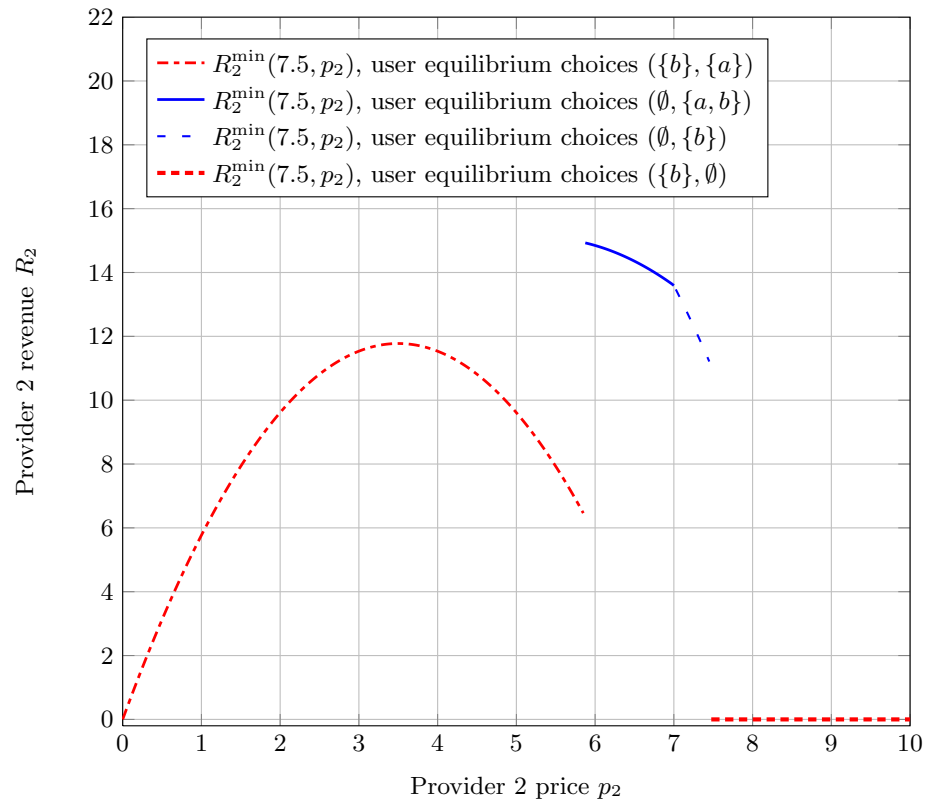

Fig. 4. Minimal revenue $R_{2}^{\min }\left(7.5, p_{2}\right)$ in terms of $p_{2}$, with segment choices at Wardrop equilibrium.

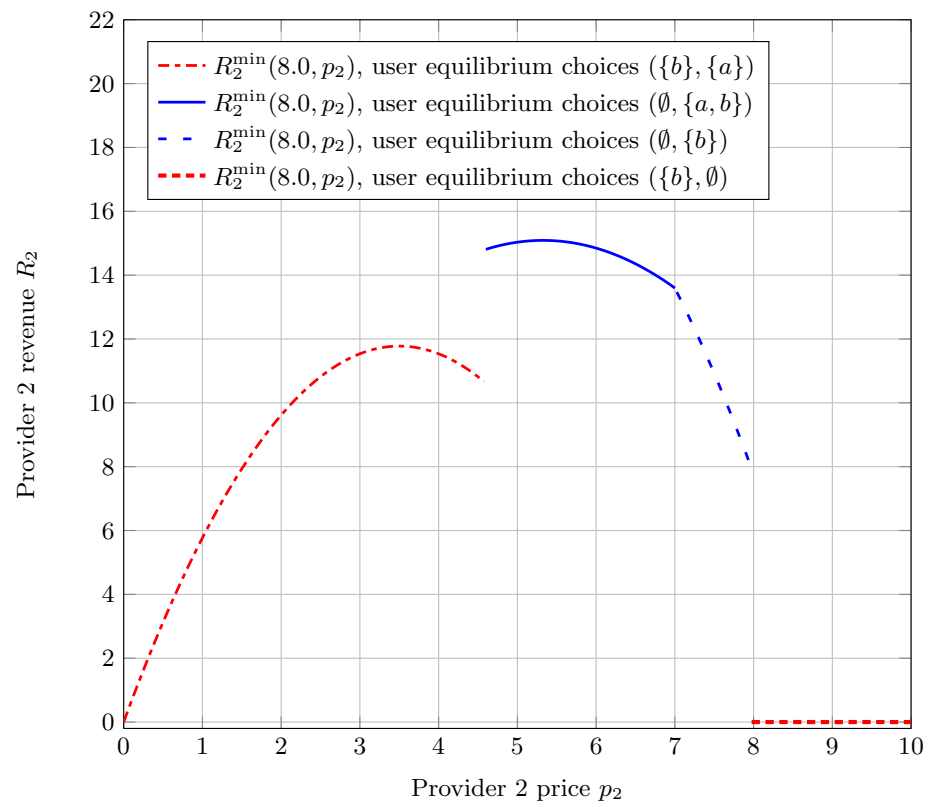

Fig. 5. Minimal revenue $R_{2}^{\min }\left(8.0, p_{2}\right)$ in terms of $p_{2}$, with segment choices at Wardrop equilibrium. 
Interestingly, we observe that the set of segments that an operator attracts is not necessarily decreasing in its price, a counter-intuitive phenomenon. On Figure 5, Operator 2 attracts Segment $b$ only when its price is above 4.5. This can be explained as follows: when $p_{2}<4.5$, Segment $a$ (resp. $b$ ) prefers Operator 2 (resp. 1), and an increase in $p_{2}$ leads to a decrease in demand $d_{2, a}$. Therefore congestion decreases, and since $\gamma_{1, b}<\gamma_{2, b}$, the perceived congestion $\left(\gamma_{\cdot, b} \ell(\cdot)\right)$ for Segment $b$ decreases faster on Operator 2 than on Operator 1 . In our instance, that congestion cost loss exceeds the price increase, so that $\bar{p}_{2, b}$ decreases faster than $\bar{p}_{1, b}$ and Segment $b$ ends up preferring Operator 2.

\section{Conclusions}

A two-level (i.e., two time scales) price competition model between wireless providers has been presented, with several segments of customers and several operators. At the shortest time scale, users of each segment select the provider offering the best compromise between price, reputation and congestion. At the largest time scale, providers compete on the price they propose, to maximize the worst-case revenue they can earn from a user equilibrium. We illustrate this model by an example of two providers competing on a two-segment market where the price equilibrium is reached with one segment per provider.

We can extend this model by introducing a multi-channel ressource-shared price competition model and studying how attributing a segment to one or more channels may influence possible price equilibria and the social welfare. Another possible extention is a more complete model partially inspired by [11] on investments in technologies and infrastructures, including our present assumption that market is segmented. This should yield investment of several providers on technologies with a shared spectrum, a result that was not possible in [11].

\section{Acknowledgment}

This work was supported by the French research agency through the CAPTURES project.

\section{References}

1. Osborne, M.J., Rubinstein, A.: A Course in Game Theory. MIT Press (1994)

2. Wardrop, J.: Some theoretical aspects of road traffic research. Proc. of the Institute of Civil Engineers 1 (1952) 325-378

3. Acemoglu, D., Ozdaglar, A.: Competition and efficiency in congested markets. Mathematics of Operations Research 32(1) (2007) 1-31

4. Acemoglu, D., Ozdaglar, A.: Price competition in communication networks. In: Proc. of IEEE INFOCOM, Barcelona, Spain (2006)

5. Bernstein, F., Federgruen, A.: A general equilibrium model for industries with price and service competition. Operations Research 52(6) (2004) 868-886 
6. Maillé, P., Tuffin, B.: Competition among providers in loss networks. Annals of Operations Research (2011) forthcoming.

7. Maillé, P., Tuffin, B.: Price war with partial spectrum sharing for competitive wireless service providers. In: Proc. of IEEE Globecom. (2009)

8. He, Q., Wu, D., Khosla, P.: SORI: a secure and objective reputation-based incentive scheme for ad-hoc networks. In: Proc. of IEEE Wireless Communications and Networking Conference (WCNC). (2004)

9. Jaramillo, J., Srikant, R.: A game theory based reputation mechanism to incentivize cooperation in wireless ad hoc networks. Ad Hoc Networks 8 (2010) 416-429

10. Ding, Q., Zhang, X., Li, X., Zhou, X.: Reputation based access point selection in 802.11 network. In: Proc. of the 3rd 2008 International Conference on Convergence and Hybrid Information Technology. (2008)

11. Maillé, P., Tuffin, B., Vigne, J.M.: Technological investment games among wireless telecommunications service providers. International Journal of Network Management 21 (2011) 65-82

12. Aashtiani, H.Z., Magnanti, T.L.: Equilibria on a congested transportation network. SIAM Journal of Algebraic and Discrete Methods 2 (1981) 213-226 